\title{
Simulations of Ground-Penetrating Radars Over Lossy and Heterogeneous Grounds
}

\author{
Levent Gürel, Senior Member, IEEE, and Uğur Oğuz
}

\begin{abstract}
The versatility of the three-dimensional (3-D) finitedifference time-domain (FDTD) method to model arbitrarily inhomogeneous geometries is exploited to simulate realistic groundpenetrating radar (GPR) scenarios for the purpose of assisting the subsequent designs of high-performance GPR hardware and software. The buried targets are modeled by conducting and dielectric prisms and disks. The ground model is implemented as lossy with surface roughness, and containing numerous inhomogeneities of arbitrary permittivities, conductivities, sizes, and locations. The impact of such an inhomogeneous ground model on the GPR signal is demonstrated. A simple detection algorithm is introduced and used to process these GPR signals. In addition to the transmitting and receiving antennas, the GPR unit is modeled with conducting and absorbing shield walls, which are employed to reduce the direct coupling to the receiver. Perfectly matched layer absorbing boundary condition is used for both simulating the physical absorbers inside the FDTD computational domain and terminating the lossy and layered background medium at the borders.
\end{abstract}

Index Terms-Finite-difference time-domain (FDTD), groundpenetrating radar (GPR), subsurface scattering.

\section{INTRODUCTION}

$\mathbf{O}$ NE PURPOSE of performing ground-penetrating radar (GPR) simulations is to simultaneously develop novel GPR configurations and detection algorithms for practical applications [1]-[3]. Results of GPR simulations can be used for this purpose. However, it should be anticipated that the effectiveness of the GPR hardware and detection algorithms in real-life applications will be limited by how accurately the simulations can model the actual GPR environment. Therefore, this paper focuses on the electromagnetic simulations of realistic GPR scenarios.

Detection algorithms are developed and used to discriminate between the buried targets and other inhomogeneities embedded in the ground. Hence, if the simulation results are to be used in the development of the detection algorithms, the heterogeneous nature of the ground should be included in the simulated model, in addition to the other details of the GPR environment. Because of its flexibility in modeling arbitrary inhomogeneities and nonuniformly layered media, the three-dimensional (3-D) finite-difference time-domain (FDTD) method [4] is chosen to simulate the GPR problem in this paper. Indeed, the FDTD method has proven to be one of the most popular techniques reported in the literature to simulate GPR problems. In

Manuscript received June 7, 2000; revised December 8, 2000.

The authors are with the Department of Electrical and Electronics Engineering, Bilkent University, TR-06533 Bilkent, Ankara, Turkey (e-mail: lgurel@ee.bilkent.edu.tr).

Publisher Item Identifier S 0196-2892(01)04838-0.

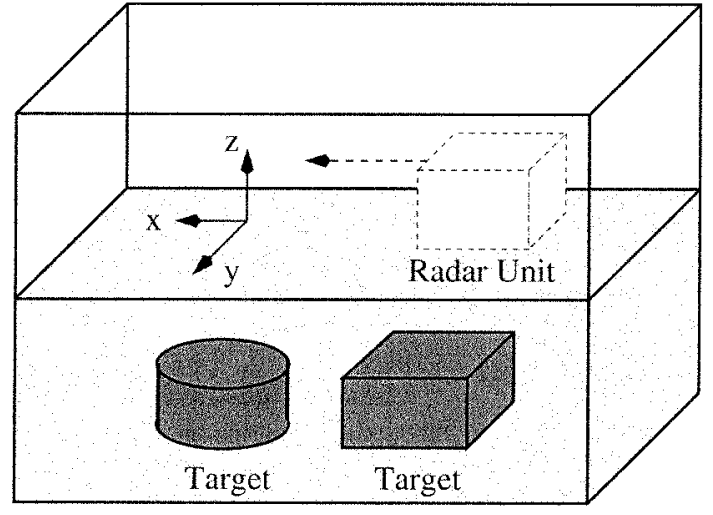

Fig. 1. Geometry of a subsurface-scattering problem with buried scatterers. A radar unit travels over the interface at a fixed elevation.

most of those reports, the ground is modeled as a homogeneous, conductive, and dielectric medium [5]-[12].

In this work, results of GPR simulations including more realistic ground models are presented, where the ground models are implemented to contain loss, surface roughness, and an arbitrary number of inhomogeneities of randomly assigned permittivities, conductivities, sizes, and locations. The details of such realistic ground models are given in Section III.

As shown in Fig. 1, the radar unit and the target(s) are also important elements of a GPR problem. Therefore, their models have to be as realistic as the ground model, too. The radar unit contains transmitting and receiving antennas that are isolated from each other by conducting shields coated with absorbers [9], [10]. The perfectly matched layer (PML) absorbing boundary condition (ABC) [13]-[16] is used to simulate these physical absorbers inside the FDTD computational domain. The details of the radar unit are described in Section II. The PML ABC is also utilized to terminate the FDTD computational domain at the borders by matching all layers of background [8], [17], be it lossy or lossless. The buried targets are modeled as conducting and dielectric prisms and disks of arbitrary sizes, permittivities, and conductivities. The air is modeled as free space (vacuum).

\section{RADAR UNIT}

The simple GPR unit depicted in Fig. 2 contains a transmitting and a receiving antenna. The GPR unit travels above the ground-air interface, at a fixed elevation. The transmitter (T) generates the fields, which propagate toward and penetrate the ground with a particular polarization. The receiver (R) collects and samples the fields with the same polarization. 


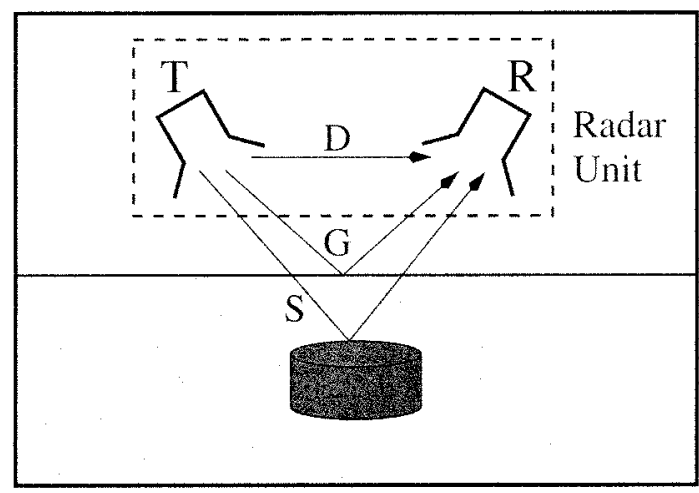

Fig. 2. Transmitter-receiver configuration of the radar unit. The total received signal is an aggregate of three signals: the direct signal (D) coupled from the transmitter to the receiver, the signal reflected from the ground $(\mathrm{G})$, and the signal scattered by the buried target $(\mathrm{S})$.

The transmitting antenna selected in this work is an $x$-polarized dipole, modeled by a single Yee cube of constant current density. The time variation of this current source is given by [14]

$$
J(t)=\frac{1}{\Delta^{3}}\left[4\left(\frac{t}{\tau}\right)^{3}-\left(\frac{t}{\tau}\right)^{4}\right] e^{-(t / \tau)}
$$

where $\tau=1 /\left(4 \pi f_{0}\right), f_{0}$ is the center frequency of the pulse, and $\Delta=\Delta x=\Delta y=\Delta z$ is the sampling interval in space. This source function is preferred for its very small dc content and its time- and bandlimited character. For all of the simulation results presented in this paper, $f_{0}=500 \mathrm{MHz}$, and the sampling intervals in space and time are selected as $\Delta=5 \mathrm{~mm}$ and $\Delta t=$ $9 \mathrm{ps}$, respectively.

The receiver is also modeled as a small dipole that samples the $x$ component of the electric-field function $E_{x}(x, y, z, t)$ with a sampling period of $\Delta t$ in time. When the radar unit is stationary and the receiver collects data at a point $\left(x_{0}, y_{0}, z_{0}\right)$ in space for successive instants of time, this is called an A-scan, and the resulting data is denoted as

$$
E_{x}^{n}=E_{x}\left(i \Delta x=x_{0}, j \Delta y=y_{0}, k \Delta z=z_{0}, n \Delta t\right) .
$$

A B-scan is obtained by performing repeated A-scan measurements at discrete points on a linear path, as illustrated in Fig. 1. For example, if the radar unit moves in the $x$ direction, the collected B-scan data is denoted as

$$
E_{x}^{i, n}=E_{x}\left(i \Delta x, j \Delta y=y_{0}, k \Delta z=z_{0}, n \Delta t\right) .
$$

Similarly

$$
E_{x}^{i, j, n}=E_{x}\left(i \Delta x, j \Delta y, k \Delta z=z_{0}, n \Delta t\right)
$$

denotes the data collected on a rectangular grid of discrete points on a constant $z$ plane. This measurement is called a C-scan and can be considered as combining several B-scans.

In the transmitter-receiver (TR) configuration, the receiving antenna collects not only the fields scattered from the target (S), but also the direct signal coupled from the transmitter (D), and

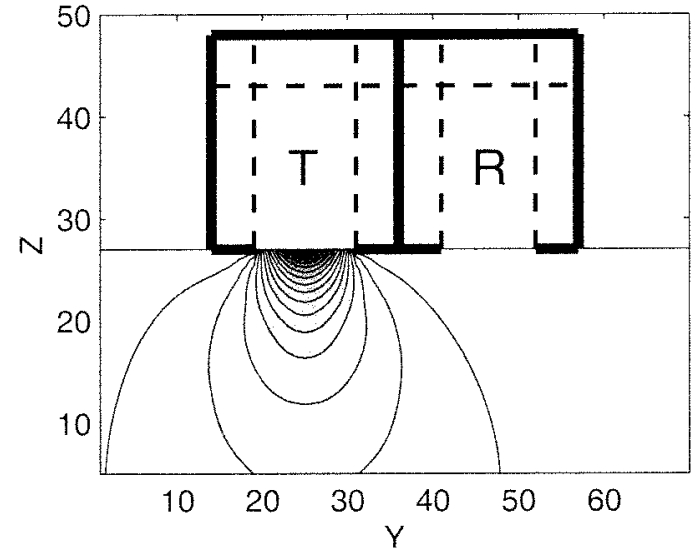

Fig. 3. Geometry and the near-field radiation pattern on the shield model with inner walls coated by PML absorbers.

the signal reflected from the ground $(\mathrm{G})$. The main problem faced in the TR configuration is that the D signal is too large with respect to the desired $\mathrm{S}$ signal.

When the $\mathrm{D}, \mathrm{G}$, and $\mathrm{S}$ signals are superposed at the receiver location to make up the total signal $\mathrm{D}+\mathrm{G}+\mathrm{S}$, the energy of the $\mathrm{S}$ signal constitutes a very small portion of the total received energy. Therefore, it is quite hard to detect the scattered signal in the total received signal. In this paper, in order to overcome this difficulty, the transmitting and the receiving antennas are isolated using conducting walls supported by PML absorbers [9], [10]. These shield walls enclose the two antennas in two chambers, leaving only the bottom faces of the chambers open, as displayed in Fig. 3. Using bare conducting walls yields large and slowly-decaying oscillations due to the resonance effects. In practice, these resonance effects are prevented by mounting high-frequency absorbers on the inner walls of shield walls. However, FDTD simulations of such novel materials become computationally expensive since these high-performance absorbers maintain rapidly decaying electromagnetic fields in a small thickness. Therefore, the inner faces of the conducting shield walls are coated with four-cell-thick PML absorbers. That is, such high-performance physical absorbers are simulated using the PML ABC in this paper. Note that the sides of the PML absorbers must also be covered by conducting shields, in order not to violate the electromagnetic boundary conditions on these side surfaces.

Fig. 3 displays the geometry of the GPR model, as well as its near-field pattern, obtained by the observation of the maximum values of the $x$ component of the electric field on a $y-z$ plane. Although the radiation pattern of a small dipole is isotropic around its axis, Fig. 3 demonstrates that the chamber model maintains good directivity toward the ground. This directivity reduces the coupling to the receiver, enhancing the detectability of the scattered signals. From this point of view, the small dipole placed in a conducting chamber, with PML ABCs mounted on the inner walls, can be regarded as a new antenna, designed especially for computational studies. This new antenna simulates practical GPR antennas with near-field patterns similar to the one shown in Fig. 3. More information about this novel GPR model can be found in [9] and [10]. 


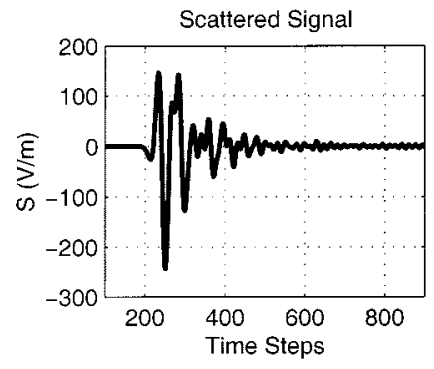

(a)

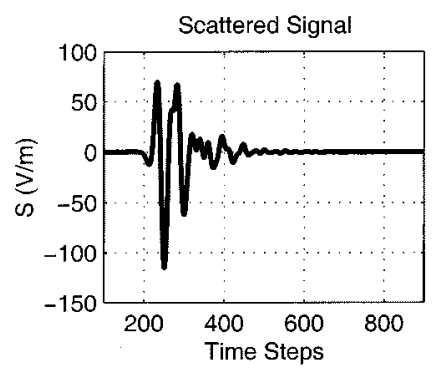

(c)

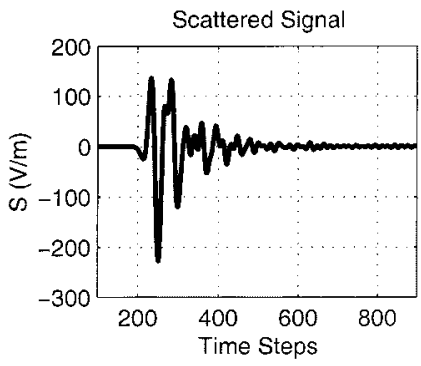

(b)

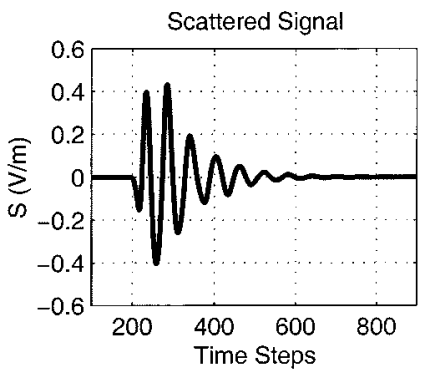

(d)
Fig. 4. Scattered signals from a conducting prism buried $5 \mathrm{~cm}$ under four lossy ground models with conductivities (a) $0.001 \mathrm{~S} / \mathrm{m}$, (b) $\mathrm{S} / \mathrm{m}$, (c) $0.1 \mathrm{~S} / \mathrm{m}$, and (d) $1.0 \mathrm{~S} / \mathrm{m}$.

\section{REALISTIC GROUND MODELS}

In this section, the shielded GPR configuration introduced in the previous section is used in the simulations of various scenarios involving realistic ground models. In all of the following simulations, the center frequency of the source is fixed at 500 $\mathrm{MHz}$ and the permittivity of the ground is $8 \epsilon_{0}$. Conductivity, heterogeneity, and the surface roughness of the ground are investigated as parameters affecting the detection of the buried target.

\section{A. Conductive Ground Models}

One main difficulty that practical GPR systems face is the humidity of the soil. Since wet soils are lossy, they are modeled by conductive media in the FDTD method. The conductivity of the ground affects both $\mathrm{G}$ and $\mathrm{S}$ signals. The higher the conductivity, the larger the reflected signal from the ground-air interface. In addition to increasing the reflectivity of the interface, higher conductivity of the ground increases the loss, which, in turn, diminishes the S signal propagating down and up in the lossy ground. Moreover, a larger $\mathrm{G}$ signal contributes to the longer tail of the total received signal. All of these effects make it harder to detect the $\mathrm{S}$ signal in the total received signal, and thus, to detect the buried targets in wet soils. However, the more important impact of the ground conductivity is on the $\mathrm{S}$ signal, which is the signal scattered from the target.

The decay of the $\mathrm{S}$ signal in lossy grounds is demonstrated by four simulations, with results shown in Fig. 4(a)-(d). In these simulations, conducting prisms of size $5 \times 5 \times 4 \mathrm{~cm}^{3}$ are buried $5 \mathrm{~cm}$ under lossy ground models with conductivities $0.001 \mathrm{~S} / \mathrm{m}, 0.01 \mathrm{~S} / \mathrm{m}, 0.1 \mathrm{~S} / \mathrm{m}$, and 1.0 S/m. Fig. 4(a) and (b) demonstrate that the conductivity values $0.001 \mathrm{~S} / \mathrm{m}$ and 0.01

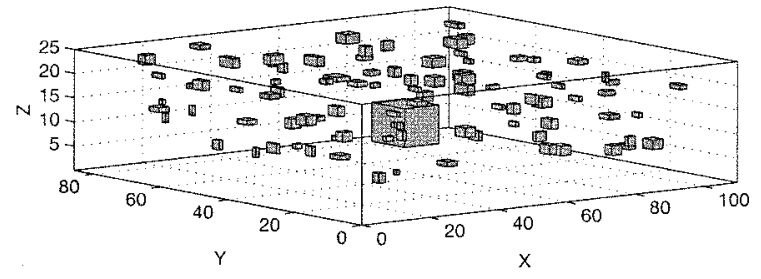

(a)

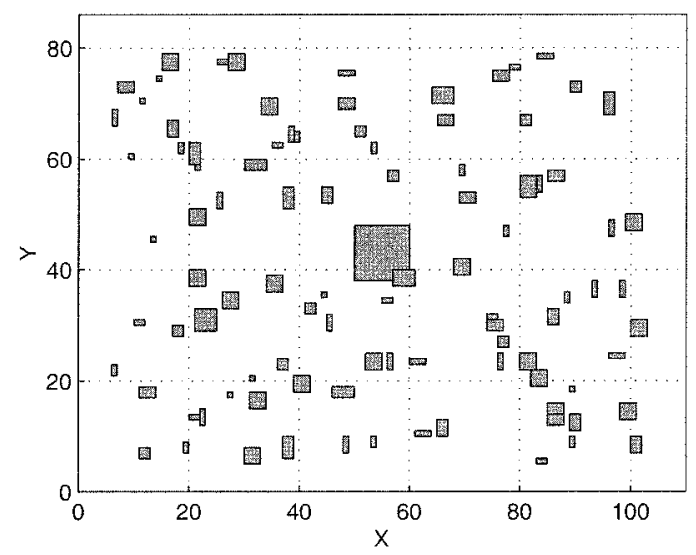

(b)

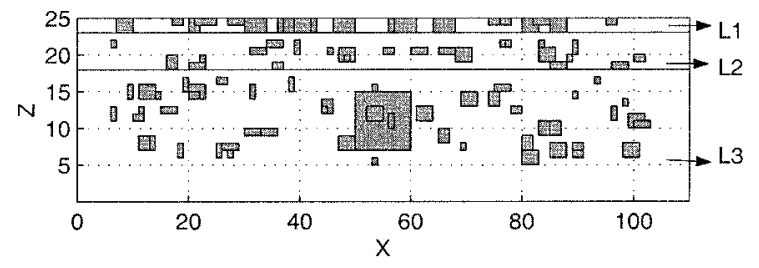

(c)

Fig. 5. First heterogeneous ground model, with a conductivity value of $0.01 \mathrm{~S} / \mathrm{m}$ and a permittivity value of $8 \epsilon_{0}$. There are 20 holes on the surface of the ground. The second ground level contains 25 highly conducting inhomogeneities and the third level contains 50 scatterers with relatively lower conductivity values. The target is buried $5 \mathrm{~cm}$ deep.

$\mathrm{S} / \mathrm{m}$ do not cause any major attenuation on the scattered signals. However, the amplitudes of the scattered signals decrease as the ground conductivity values increase up to $0.1 \mathrm{~S} / \mathrm{m}$ and 1.0 $\mathrm{S} / \mathrm{m}$, as shown in Fig. 4(c) and (d). Conductivity values below $0.1 \mathrm{~S} / \mathrm{m}$ may be regarded as low values for GPR scenarios at this center frequency $(500 \mathrm{MHz})$.

\section{B. Heterogeneous Ground Models}

An invariable feature of real-life soils is heterogeneity, which should be represented in a realistic ground model. Without using the simulation results containing some inhomogeneities altering the idealized nature of the ground model, it becomes a futile effort to develop a meaningful detection algorithm that will perform well over real-life soils.

An example of a realistic ground model is depicted in Fig. 5. This model is formed by simulating small scatterers embedded in the ground. The sizes, locations, and material properties of these inhomogeneities are determined randomly within preset 
limits. For example, the maximum dimensions of the inhomogeneities are $4 \Delta(2 \mathrm{~cm}), 4 \Delta(2 \mathrm{~cm})$, and $2 \Delta(1 \mathrm{~cm})$ in the $x$, $y$, and $z$ directions, respectively. The cross-sectional view of Fig. 5(c) shows that three different types of inhomogeneities are placed in three separate levels of the ground. In the first level (L1), surface roughness is simulated by creating 20 small holes. These holes are implemented by selecting the permittivity of the randomly distributed boxes as $\epsilon_{0}$. In the second level (L2), a part of the ground that is assumed to host 25 relatively more conductive objects, e.g., plant roots, is simulated. The thickness of L2 is $5 \Delta(2.5 \mathrm{~cm})$. The third and the deepest level (L3) contains the actual target surrounded by 50 scatterers with relatively lower conductivities. All inhomogeneities in L2 and L3 have randomly selected permittivity values between $\epsilon_{0}$ and $16 \epsilon_{0}$. The ranges of random conductivities of the scatterers in L2 and L3 are 0.1/0.2 $\mathrm{S} / \mathrm{m}$ and $0.03 / 0.04 \mathrm{~S} / \mathrm{m}$, respectively.

\section{Simulation Results of Heterogeneous Ground Models}

The ground model illustrated in Fig. 5 is used in two simulations, whose unprocessed (raw) B-scan results are given in Fig. 6. In both simulations, the radar unit travels above the ground at an elevation of $2.5 \mathrm{~cm}$, and the target is buried 5 $\mathrm{cm}$ under the ground. Fig. 6(a) and (b) display raw data of total received signals obtained in the two simulations, where the targets are selected as a perfectly conducting disk and a dielectric disk with permittivity $3 \epsilon_{0}$, respectively. Both disks, which have diameters of $10 \Delta(5 \mathrm{~cm})$ and heights of $8 \Delta(4 \mathrm{~cm})$, are accurately modeled using the contour path method [18]. The ground model has a conductivity of $0.01 \mathrm{~S} / \mathrm{m}$ and a permittivity of $8 \epsilon_{0}$. Both simulation results are given as gray-scale images. The horizontal axes in these images represent the radar position, while the vertical axes represent the time steps $(\Delta t)$, which can also be interpreted as the depth into the ground. For each target, the maxima of the B-scan electric-field values $E_{x}^{i, n}$ are displayed in the title of the corresponding plot.

Additionally, two energy plots are given with each B-scan image in Fig. 6(a) and (b). The first of these plots is the one below the B-scan image, displaying the cumulative energy received at each GPR position on the path. The displayed energy values are computed by summing the square of the normalized electric-field values observed at each time step of the corresponding A-scan, that is

$$
\text { Energy }^{i}=\sum_{n}\left|\frac{E_{x}^{i, n}}{\max _{i, n}\left(E_{x}^{i, n}\right)}\right|^{2}
$$

where the normalization factor is the maximum of the B-scan electric-field data. The second energy plot is placed to the right of the B-scan image. This plot displays the summation of the square of the discrete normalized electric-field values observed at a particular time step of each A-scan, given by

$$
\operatorname{Energy}^{n}=\sum_{i}\left|\frac{E_{x}^{i, n}}{\max _{i, n}\left(E_{x}^{i, n}\right)}\right|^{2}
$$

The summation in (6) can also be considered as the cumulative energy at a particular level of the ground. The plots of (5)

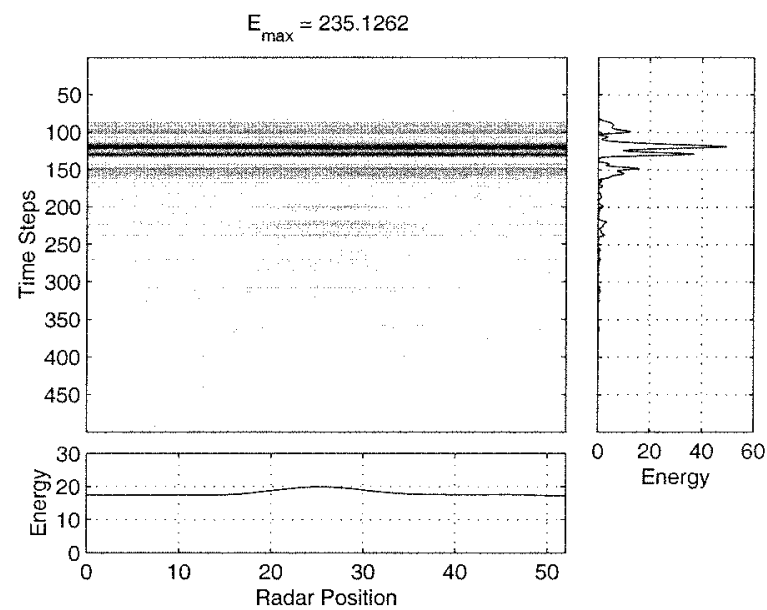

(a)

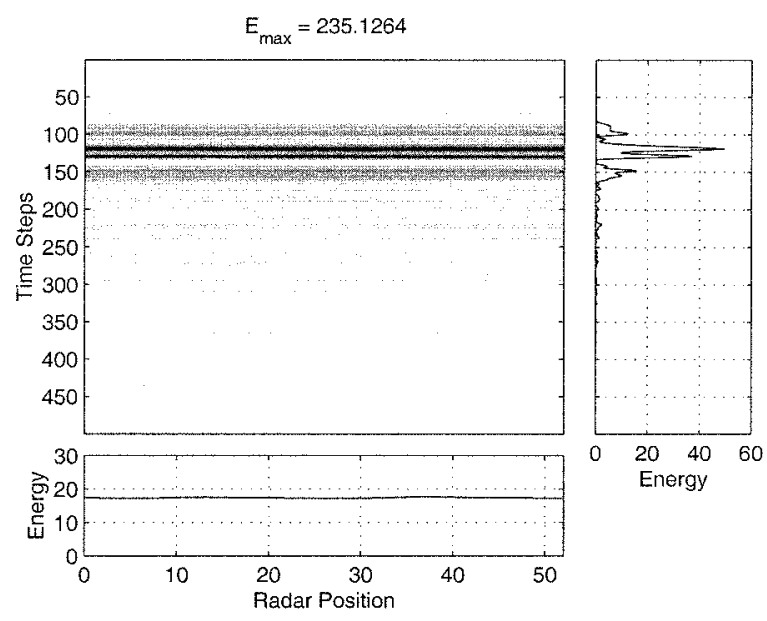

(b)

Fig. 6. Unprocessed simulation results of (a) a perfectly conducting disk and (b) a dielectric disk with permittivity $3 \epsilon_{0}$, buried $5 \mathrm{~cm}$ in the ground model of Fig. 5.

and (6) are, respectively, named as constant-position and constant-depth energy plots in this work.

Comparison of Fig. 6(a) and (b) reveals the following points.

- The reflection from the ground surface dominates the raw data, forming a black strip between the time steps 100 and 150.

- The conducting disk produces a slightly higher reflection than the dielectric disk, and the signals reflected from it can be observed in Fig. 6(a) between time steps 200 and 300 and locations $20 \Delta$ and $30 \Delta$.

- The same visual detection of the conducting disk can also be made on the constant-position energy plot, which displays a hint of a local maximum corresponding to the buried target, when the GPR unit is between locations $20 \Delta$ and $30 \Delta$.

- It is not possible to visually detect the dielectric disk from the raw data, as apparent from Fig. 6(b).

- The cumulative energy at the ground-air interface, represented by the maxima in each constant-depth plot, are highly dominant, and it is hard to visually detect either one of the buried targets from these plots. 


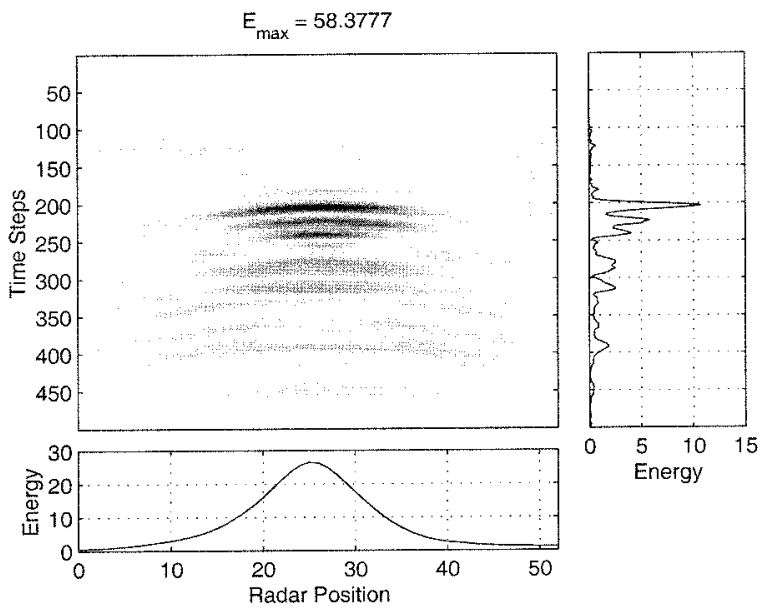

(a)

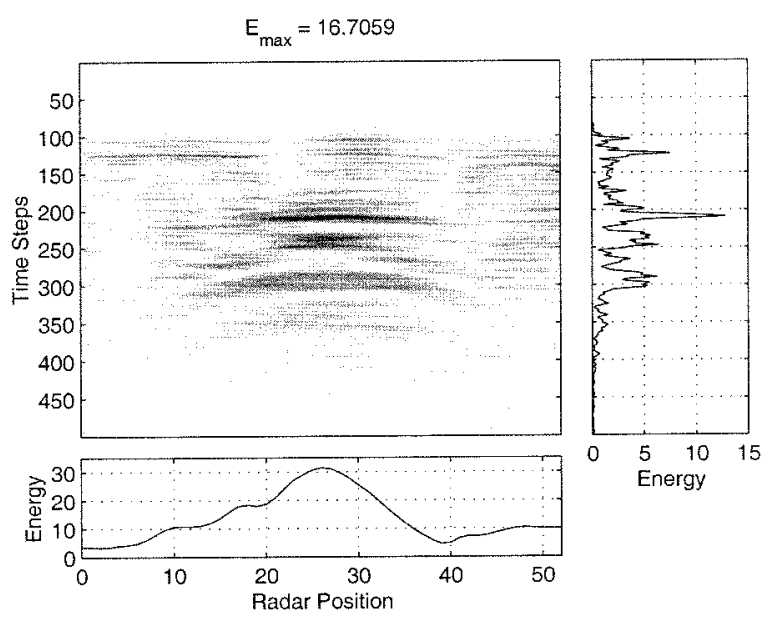

(b)

Fig. 7. Processed simulation results of (a) a perfectly conducting disk and (b) a dielectric disk with permittivity $3 \epsilon_{0}$, buried $5 \mathrm{~cm}$ in the ground model of Fig. 5 .

\section{Simple Detection Algorithm}

The results of the previous section demonstrate that, even though the shielded GPR configuration is used to boost the $\mathrm{S}$ signal with respect to the $\mathrm{D}+\mathrm{G}$ signal, visual detection of the target in the raw data is not always possible. This may be due to various reasons, such as the depth of the target, the loss of the ground, or the surface roughness of the ground-air interface. Since these practical situations can always be possible, well-designed GPR systems almost never rely on visual detection. Instead, they employ detection algorithms implemented in the software with the purpose of amplifying and isolating the $S$ signal and suppressing the $\mathrm{D}+\mathrm{G}$ signal as much as possible, much like the shielded GPR configuration aims to achieve the same in the hardware.

The perfect detection algorithm should completely subtract the $\mathrm{D}+\mathrm{G}$ signal from the total received signal to obtain the cleanest possible $\mathrm{S}$ signal. However, it is not possible to obtain the $\mathrm{D}+\mathrm{G}$ signal for every practical ground sample, especially when the ground is inhomogeneous. An alternative is to opt for an imperfect detection algorithm that will approximate

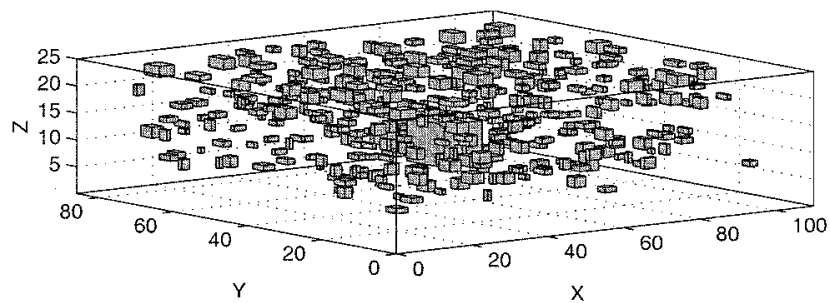

(a)

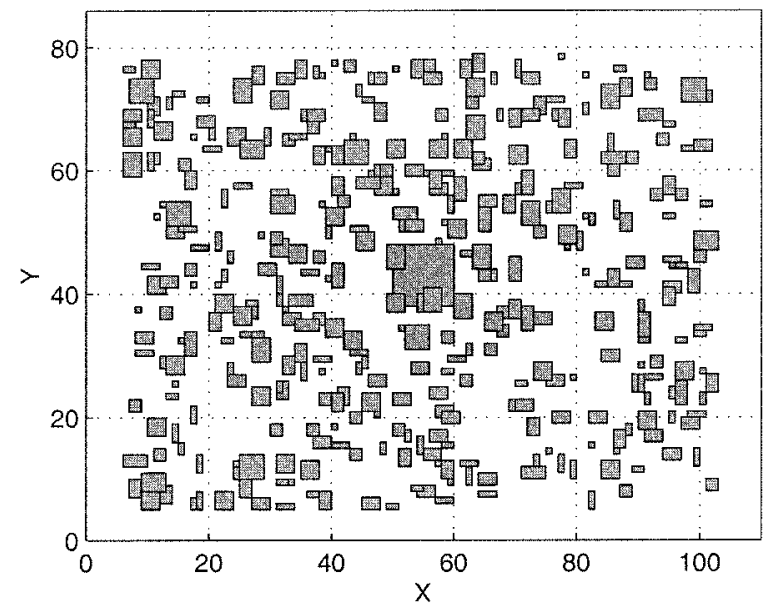

(b)

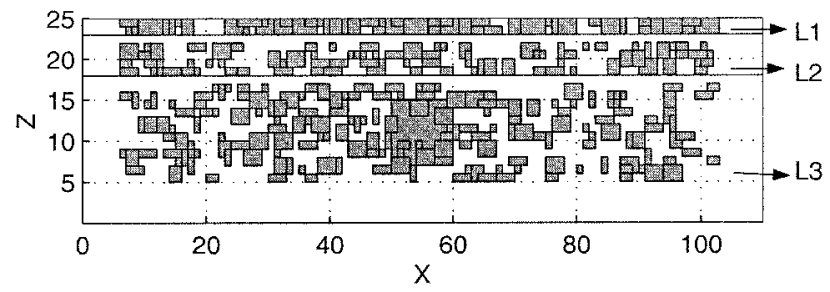

(c)

Fig. 8. Second heterogeneous ground model. There are 80 holes on the ground surface, 100 highly lossy scatterers in the second layer, and 200 slightly lossy small scatterers in the third layer. The target is buried $5 \mathrm{~cm}$ under the ground-air interface. The ground has a conductivity of $0.01 \mathrm{~S} / \mathrm{m}$ and a permittivity of $8 \epsilon_{0}$.

the $\mathrm{D}+\mathrm{G}$ signal reasonably accurately even for heterogeneous soils. One of the simpler versions of such an algorithm can be developed by approximating the $\mathrm{D}+\mathrm{G}$ signal through an averaging process. Assuming that an area of a representative sample of the ground located away from the target, a C-scan can be performed over this area. Since the ground is not uniform, each A-scan signal constituting the $\mathrm{C}$-scan data should be different. The recorded signals can be averaged into a single reference signal obtained by

$$
\tilde{E_{x}^{n}}=\sum_{i, j} \frac{E_{x}^{i, j, n}}{i_{\max } j_{\max }} .
$$

The denominator $i_{\max } j_{\max }$ in (7) is the number of A-scan signals included in the averaging. Once this reference signal is obtained as an approximation to the $\mathrm{D}+\mathrm{G}$ signal, it can be 


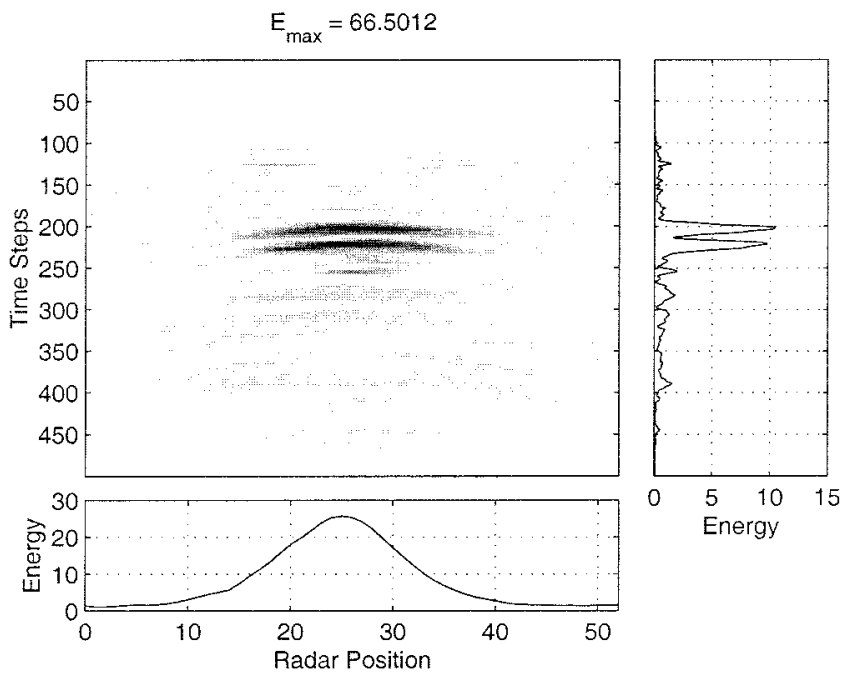

(a)

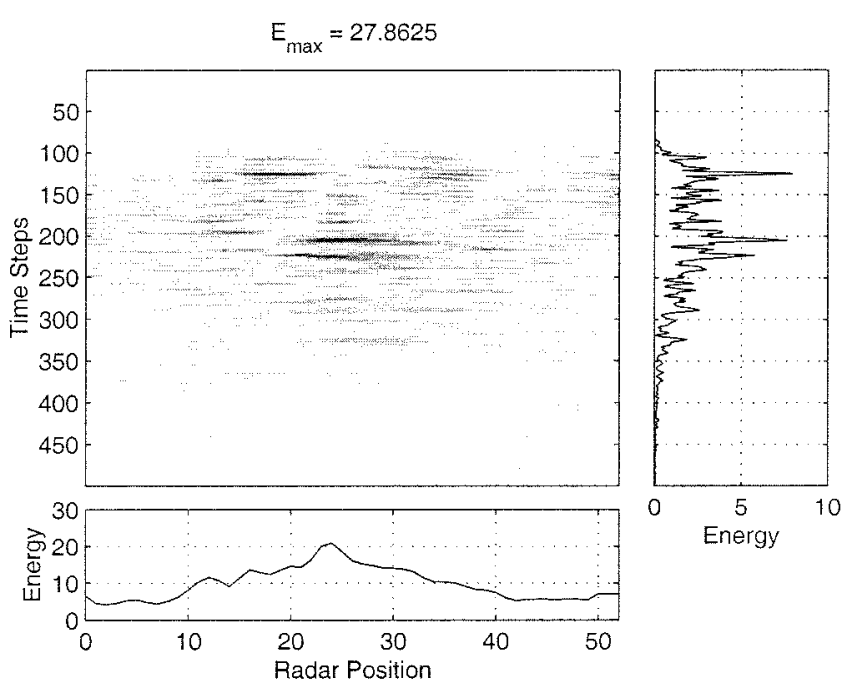

(b)

Fig. 9. Processed simulation results of (a) a perfectly conducting disk and (b) a dielectric disk with permittivity $3 \epsilon_{0}$, buried $5 \mathrm{~cm}$ in the ground model of Fig. 8 .

subtracted from any A-scan signal to approximate the S signal. The resultant difference signal

$$
\bar{E}_{x}^{n}=E_{x}^{n}-\tilde{E}_{x}^{n}
$$

is completely free from the $\mathrm{D}$ signal and does not contain most of the $\mathrm{G}$ signal. It is possible to develop more sophisticated detection algorithms. However, this simple algorithm is sufficient for the purposes of this paper.

\section{E. Simulation Results with Simple Detection Algorithm}

Applying the aforementioned detection algorithm to each A-scan signal in Fig. 6(a) and (b), the resultant B-scan images and energy plots given in Fig. 7(a) and (b) are obtained for the conducting and dielectric disks, respectively. Comparison of the processed and unprocessed simulation results of the conducting disk (buried under the ground model of Fig. 5) clearly reveals that the simple detection algorithm successfully removes most

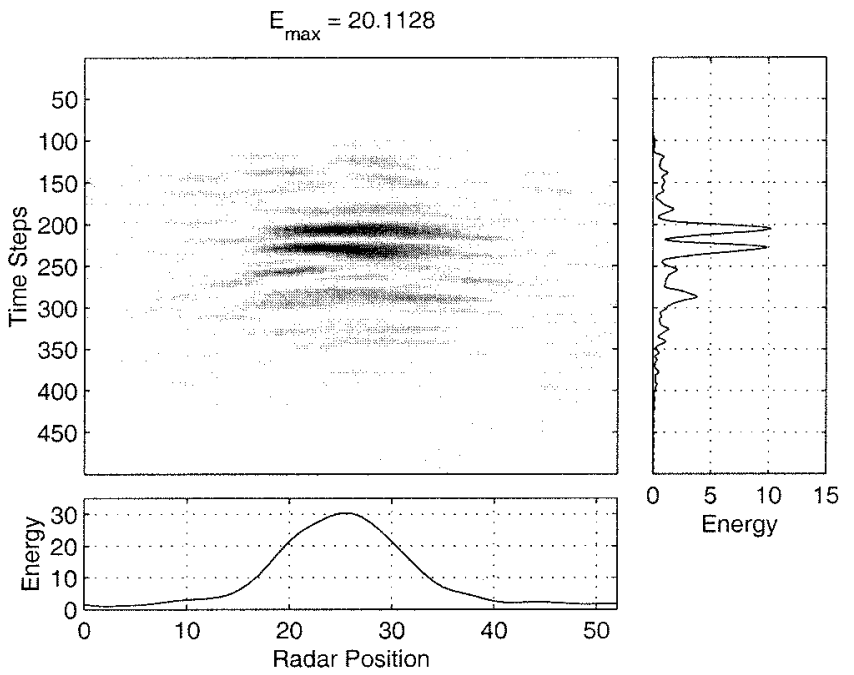

Fig. 10. Processed simulation results of a dielectric disk buried $5 \mathrm{~cm}$ in a heterogeneous ground model that is almost the same as in Fig. 8 but with no holes on the surface.

of the D and G signals from the total signal. While it is hard to visually detect the scattered signals on the B-scan image given in Fig. 6(a), the same signals are apparent in the processed B-scan image shown in Fig. 7(a). The amplitude of the removed signal can be estimated by comparing the maximum values observed in the raw and processed data, given in the titles of the two B-scan images of Figs. 6(a) and 7(a). The position and depth of the buried target can be precisely deduced from the maxima in the cumulative energy plots of Fig. 7(a).

The impact of the simple algorithm is better illustrated comparing the raw and the processed data obtained from the simulation of the dielectric disk. Although it is not at all possible to visualize the dielectric disk from the raw data images displayed in Fig. 6(b), the simple algorithm makes the detection of the dielectric disk possible, as shown in Fig. 7(b). The signals scattered from the target produce clear peaks in the correct radar-position and depth values of the cumulative energy plots. The scattered energy is smaller compared to the energy scattered from the conducting disk, causing the peak corresponding to the ground-air interface in the constant-depth plot appear higher.

In order to make the ground model even more realistic and test the detection algorithm under more difficult circumstances, the number of inhomogeneities in the heterogeneous ground model of Fig. 5 is quadrupled to reach the geometry illustrated in Fig. 8. This model contains 80 holes in L1, 100 highly lossy scatterers in L2, and 200 slightly lossy inhomogeneities in L3. Apart from four times higher density of the random holes and scatterers, all of the parameters of the geometry are the same as those of Fig. 5 .

The simulation results of a conducting disk and a dielectric disk with permittivity $3 \epsilon_{0}$, buried in the ground model of Fig. 8 , are displayed in Fig. 9(a) and (b), respectively. Fig. 9(a) demonstrates that the scattered signals from conducting disk are not affected by the highly heterogeneous ground model. The B-scan image clearly depicts and the energy plots successfully indicate the depth and position of the conducting disk. However, the same argument does not hold for the dielectric disk. The scattered signals are hardly visible in the B-scan image in Fig. 9(b). 


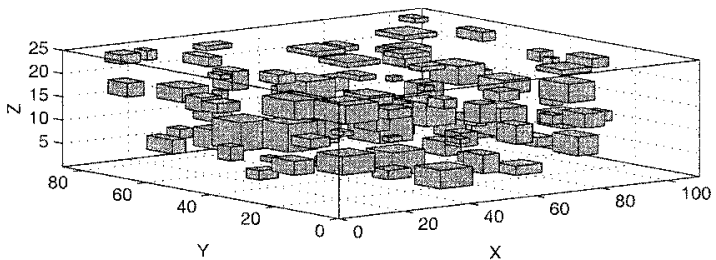

(a)

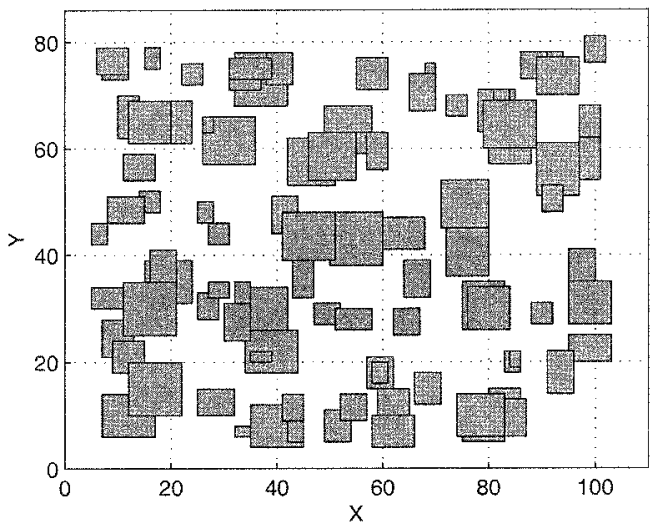

(b)

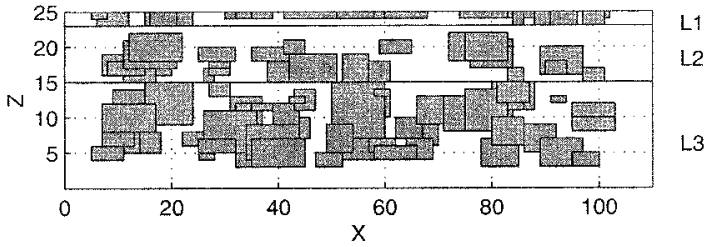

(c)

Fig. 11. Third heterogeneous ground model. There are 20 holes on the ground surface, 25 highly lossy scatterers in the second layer, and 50 slightly lossy small scatterers in the third layer. The heterogeneities are much larger compared to the small scatterers displayed in Fig. 5 . The target is buried $5 \mathrm{~cm}$ under the ground-air interface. The ground has a conductivity of $0.01 \mathrm{~S} / \mathrm{m}$ and a permittivity of $8 \epsilon_{0}$.

The energy peak in the constant-position plot is not as clear as the peak in Fig. 9(a). The peak corresponding to the inhomogeneities at the interface are larger than the peak produced by the dielectric target in the constant-depth energy plot in Fig. 9(b). Only the trained eyes would be able to visually detect the target in this very heterogeneous ground model despite the use of both shielded GPR and simple detection algorithm.

1) Ground Model with a Smooth Surface: One important observation made on the constant-depth energy plot in Fig. 9(b) is that the dominant noise caused by the inhomogeneities correspond to the ground-air interface. In order to further investigate the effect of the surface roughness, the ground model is slightly modified. The holes in the previous model are moved one cell below the ground-air interface. That is, they are no longer holes, but air bubbles under the interface, instead. Apart from this minimal modification, the number and density of the inhomogeneities and all other parameters of the ground model are kept the same. Fig. 10 shows the simulation results obtained with a dielectric disk of permittivity $3 \epsilon_{0}$ buried in this modified ground model with no surface roughness. The results displayed

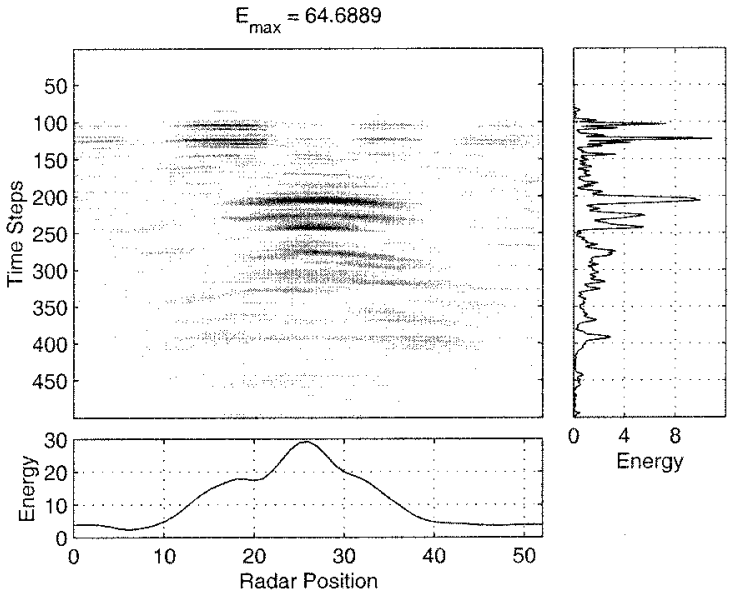

(a)

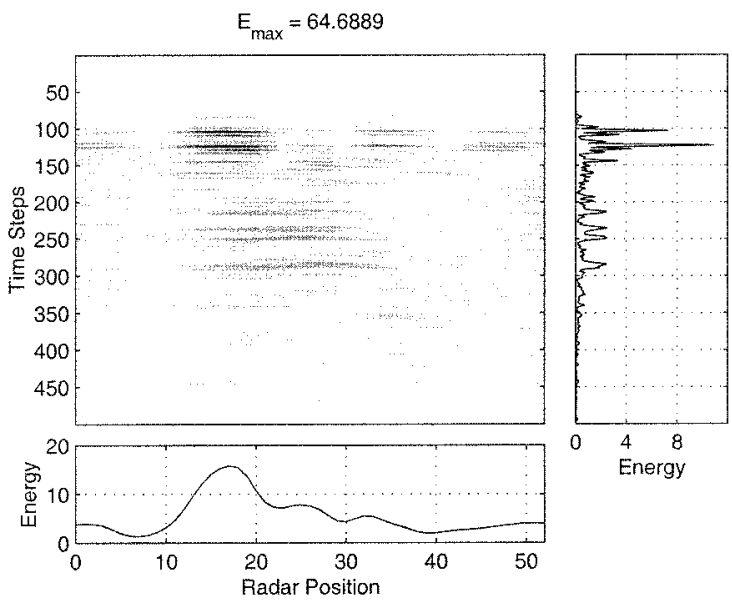

(b)

Fig. 12. Processed simulation results of (a) a perfectly conducting disk and (b) a dielectric disk with permittivity $3 \epsilon_{0}$, buried $5 \mathrm{~cm}$ in the ground model of Fig. 11.

in Fig. 10 are much better than the results given in Fig. 9(b). The scattered signals are clearly visible in the B-scan image, and the position and depth of the target can be easily deduced from the two energy plots in Fig. 10. The difference between the results displayed in Figs. 9(b) and 10 is attributed to the absence of the holes on the surface of the ground model.

2) Ground Model with Larger Heterogeneities: In order to demonstrate that the detection process is affected not only by the quantity, but also the size of the heterogeneities, the ground model in Fig. 5 is altered by substituting larger scatterers instead of the small objects. This new ground model is depicted in Fig. 11. This model contains statistically distributed surface holes (in level $L 1$ ) as wide as the main target, and dielectric objects, with dimensions comparable to those of the target, embedded in the ground. The ground model in Fig. 11 is used in two simulations, with results presented in Fig. 12(a) and (b). The first one is the simulation result of a perfectly conducting disk, identical to the one introduced in Section III-C, buried 5 $\mathrm{cm}$ under the ground-air interface. Although a large hole on the surface creates its own peaks in all three plots of Fig. 12(a) and complicates the detection, the conducting disk is still clearly 
identified in all three plots. However, for the dielectric disk results in Fig. 12(b), the reflections from the large surface holes are dominant in the overall scattered signal. Although a small peak is observed at the location of the dielectric disk in the constant-position energy plot, Fig. 12(b) does not reveal the existence of any specific buried target in the ground.

\section{CONCLUSION}

In this paper, the use and importance of realistic ground models in the 3-D FDTD simulations of GPR problems are demonstrated. Lossy ground models are employed in order to simulate the ground humidity. Inhomogeneities with arbitrary and random permittivity and conductivity values are embedded in the ground. Additionally, surface roughness is modeled by placing scatterers with $\epsilon_{0}$ permittivity and zero conductivity on the ground-air interface. The effects of loss, heterogeneity, and surface roughness of the ground are demonstrated and explained by using the results of the GPR simulations. The benefits and shortcomings of a simple detection algorithm in processing the GPR data involving heterogeneous ground models are exemplified. The humidity and heterogeneity of real-life soils should be taken into account in the design of practical GPRs and in the development of high-performance detection and fusion algorithms running on those GPRs. The simulation results presented in this paper demonstrate the main features of such realistic ground models, and therefore, can be used in subsequent design efforts.

Exploiting the flexibility of the 3-D FDTD method, the GPR unit is modeled as a TR configuration with conducting shields coated with absorbers employed to reduce the direct coupling to the receiver. The PML ABC is used to simulate these absorbers inside the FDTD computational domain. In addition, the PML $\mathrm{ABC}$ is also used at the borders of the FDTD computational domain to terminate the layered background medium, which is formed by the lossy ground and the air (vacuum). The buried objects are modeled as rectangular prisms and cylindrical disks with arbitrary conductivities and permittivities.

\section{REFERENCES}

[1] D. J. Daniels, Surface-Penetrating Radar, IEE, London, U.K., 1996.

[2] Proc. SPIE Detection and Remediation Technologies for Mines and Minelike Targets III, A. C. Dubey, J. F. Harvey, and J. T. Broach, Eds. Orlando, FL, Apr. 1998.

[3] 7th Int. Conf. Ground-Penetrating Radar. Lawrence, KS, May 1998.

[4] K. S. Yee, "Numerical solution of initial boundary value problems involving Maxwell's equations in isotropic media," IEEE Trans. Antennas Propagat., vol. AP-14, pp. 302-307, May 1966.

[5] M. Moghaddam, W. C. Chew, B. Anderson, E. J. Yannakakis, and Q. H. Liu, "Computation of transient electromagnetic waves in inhomogeneous media," Radio Sci., vol. 26, pp. 265-273, Jan.-Feb. 1991.

[6] J. M. Bourgeois and G. S. Smith, "A fully three-dimensional simulation of a ground-penetrating radar: FDTD theory compared with experiment," IEEE Trans. Geosci. Remote Sensing, vol. 34, pp. 36-44, Jan. 1996.

[7] U. Oğuz and L. Gürel, "Subsurface-scattering calculations via the 3D FDTD method employing PML ABC for layered media," in IEEE Antennas Propagation Soc. Int. Symp. URSI Radio Science Meeting, Montréal, QC, Canada, July 1997.

[8] L. Gürel and U. Oğuz, "Three-dimensional FDTD modeling of a groundpenetrating radar," IEEE Trans. Geosci. Remote Sensing, vol. 38, pp. 1513-1521, July 2000.
[9] — - "Employing PML absorbers in the design and simulation of ground penetrating radars," in 1999 IEEE IEEE Antennas Propagation Soc. Int. Symp. USNC/URSI National Radio Science Meeting, Orlando, FL, July 1999.

[10] U. Oğuz and L. Gürel, "Modeling of ground-penetrating-radar antennas with shields and simulated absorbers," IEEE Trans. Antennas Propagat., vol. 48, Nov. 2000.

[11] J. M. Bourgeois and G. S. Smith, "A complete electromagnetic simulation of the separated-aperture sensor for detecting buried land mines," IEEE Trans. Antennas Propagat., vol. 46, pp. 1419-1426, Oct. 1998.

[12] F. L. Teixeria, W. C. Chew, M. Straka, and M. L. Oristaglio, "Finite-difference time-domain simulation of ground-penetrating radar on dispersive, inhomogeneous, and conductive soils," IEEE Trans. Geosci. Remote Sensing, vol. 36, pp. 1928-1937, Nov. 1998.

[13] J. P. Berenger, "A perfectly matched layer for the absorption of electromagnetic waves," J. Comput. Phys., pp. 185-200, Oct. 1994.

[14] W. C. Chew and W. H. Weedon, "A 3D perfectly matched medium from modified Maxwell's equations with stretched coordinates," Microw. Opt. Technol. Lett., vol. 7, pp. 599-604, Sept. 1994.

[15] S. D. Gedney, "An anisotropic PML absorbing media for FDTD simulation of fields in lossy dispersive media," Electromagnetics, pp. 399-415, July/Aug. 1996.

[16] D. Johnson, C. Furse, and A. Tripp, "Application and optimization of the perfectly matched layer boundary condition for geophysical simulations," Microw. Opt. Technol. Lett., to be published.

[17] J. B. Verdu, R. Gillard, K. Moustadir, and J. Citerne, "An extension of the PML technique to the FDTD analysis of multilayer planar circuits and antennas," Microw. Opt. Technol. Lett., vol. 10, pp. 323-327, Dec. 1995.

[18] T. G. Jurgens, A. Taflove, K. Umashankar, and T. G. Moore, "Finite-difference time-domain modeling of curved surfaces," IEEE Trans. Antennas Propagat., vol. 40, pp. 357-366, Apr. 1992.

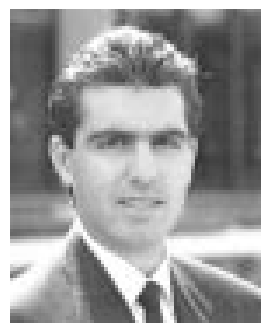

Levent Gürel (S'87-M'92-SM'97) was born in İzmir, Turkey, in 1964. He received the B.Sc. degree from the Middle East Technical University (METU), Ankara, Turkey, in 1986, and the M.S. and Ph.D. degrees from the University of Illinois, Urbana-Champaign (UIUC), in 1988 and 1991, respectively, both in electrical engineering.

He joined the Thomas J. Watson Research Center of IBM, Yorktown Heights, NY, in 1991, where he worked as a Research Staff Member on the electromagnetic compatibility (EMC) problems related to electronic packaging, on the use of microwave processes in the manufacturing and testing of electronic circuits, and on the development of fast solvers for interconnect modeling. He became an Associate Professor of the Institute of Higher Education, Ankara, in 1993. Since 1994, he has been a Faculty Member with the Department of Electrical and Electronics Engineering, Bilkent University, Ankara. He was a Visiting Associate Professor at the Center for Computational Electromagnetics (CCEM) of the UIUC for one semester in 1997. His research interests include the development of fast algorithms for computational electromagnetics (CEM) and the application thereof to scattering and radiation problems involving large and complicated scatterers, antennas and radars, frequency-selective surfaces and high-speed electronic circuits. He is also interested in the theoretical and computational aspects of electromagnetic compatibility and interference analyses. Ground-penetrating radars and other subsurface-scattering applications are also among his current research interests.

Dr. Gürel is Chairman of the AP/MTT/ED/EMC Chapter of the IEEE Turkey Section. He has published several papers in IEEE journals, actively attended numerous IEEE symposia, and served as a reviewer for various IEEE journals.

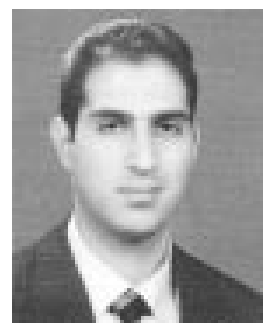

Uğur Oğuz was born in Ankara, Turkey, in 1973. He received the B.Sc. and M.Sc. degrees, both in electrical engineering, from Bilkent University, Ankara, Turkey, in 1994 and 1997, respectively.

From August 1997 to October 1998, he served in the Turkish Army as a Database Manager and Computer System Administrator. Since November 1998, he has been a Research Engineer, Department of Electrical and Electronics Engineering, Bilkent University. His research interests include time-domain methods in computational electromagnetics and their applications to geophysical problems. 